\title{
Weight Zero Enhancement In Speech Synthesis Using Neural Networks
}

\author{
G.C. Cawley, M.I. Heywood, P.D. Noakes.
}

Neural and VLSI Systems Laboratory, Department of Electronic Systems Engineering, University of Essex, Wivenhoe Park, Colchester, Essex, CO4 3SQ, United Kingdom.

\section{Introduction.}

In continuous speech the boundaries between allophones are not distinct, but are considerably blurred, an effect known as coarticulation, caused by the inertia of articulators (eg lips and tongue). Coarticulation carries little semantic content of an utterance, but we subconsciously expect to hear its effects in natural speech. This work is concerned with the training of neural networks for speech synthesis through generation of Linear Predictive Coding (LPC) parameters corresponding to the sequence of allophones. LPC attempts to find the coefficients $A_{k}$ of an all pole filter with a transfer function $H(z)$, such that the spectral properties are optimally similar to that of a segment of sampled speech.

\section{Data Pre - Processing}

Unfortunately, LPC coefficients are not suitable for training neural networks as they are highly sensitive to error. Consequently, the LPC coefficients are transformed to an equivalent Line Spectral Pair (LSP) representation [1], where LSP schemes are known to offer excellent quantisation and interpolation properties. LSP coding records the frequency of the zeros of two polynomials $P(z)$ and $Q(z)$ which are related to the predictor polynomial $A(z)$. The zeros of $P(z)$ and $Q(z)$ lie on the unit circle in the $z$ plane, this reduction in the search space allows efficient root finding methods to be employed. For the synthesis filter to be stable, the zeros of $\mathrm{P}(\mathrm{z})$ alternate with the poles of $\mathrm{Q}(\mathrm{z})$ around the unit circle. The overall spectral sensitivity of LSP parameters is less than that of PARCOR or log area ratio parameters. Furthermore, the spectral sensitivity of individual LSP parameters are uniform, whereas low order PARCOR parameters exhibit higher sensitivities.

\section{Network Training}

Heywood and Noakes [2] introduce a framework for performing pruning in feed forward neural networks, interactively with the standard back-propagation (BP) learning rule. To achieve this two extra learning parameters are introduced. One is a stability threshold which effectively identifies when the weights feeding a neuron represent 'useful' information. The second parameter indicates whether a stable weight is of significance at the neuron. For the purposes of this paper, the magnitude of 'stable' weights are merely compared to a Zero Weight threshold, and set to zero if they fall below this threshold [2]. However, there is no reason why a more stringent measure of weight contribution cannot be employed (eg product of weight and stimuli). Secondary controls to limit the number of weights introduced at any one weight update are also included.

\section{Results}

The main aim of these experiments is to determine the extent, if any, of weight redundancy and resulting generalisation improvements, when the performance is compared to that of the standard BP algorithm (MLP, single hidden layer). Simulation results indicate that significant weight redundancy is detected (25\% minimum, $90 \%$ maximum). In the case of the more important phonetic classes of vowel, nasal and plosive, where the data set is larger, increases in generalisation ability $(10 \%-18 \%)$ are returned, in spite of employing a simple magnitude type zero weight criteria (pruning is facilitated by a correction path provided via the learning algorithm).

\section{Acknowledgments.}

The authors acknowledge the funding of the United Kingdom Science and Engineering Research Council.

\section{References.}

[1] Cawley G.C., Noakes P.D. "LSP Speech Synthesis Using Backpropagation Networks", in - press, ANN - 93 Brighton, May 1993.

[2] Heywood M.I., Noakes P.D., "Simple addition to Back-Propagation Learning for Dynamic Weight Pruning, Sparse Network Extraction and Faster Learning", IEEE ICNN 93, San Francisco, Vol 2, pp $620-625$. 